\title{
L'EFFICACIA DEI DIRITTI SOCIALI NELLA SANITÀ PUBBLICA
}

\author{
CARLOS NETO, Daniel ${ }^{1}$ \\ DENDASCK, Carla ${ }^{2}$ \\ OLIVEIRA, Euzébio de ${ }^{3}$
}

CARLOS NETO, Daniel; DENDASCK, Carla; OLIVEIRA, Euzébio de. L'efficacia dei diritti sociali nella sanità pubblica. Conoscenza base scientifica multidisciplinare Magazine - Vol. 1. Anno. 1. Marzo. 2016, pp: 3-14 ISSN:0959-2448

\section{RIEPILOGO}

I diritti fondamentali sono state causa per varie discussioni negli ultimi anni, causando gli studiosi a dedicarsi per studiare sia attraverso l'aspetto sanitario, come attraverso la legge, la reale efficacia degli orientamenti delineati dalle organizzazioni. Questo articolo mira a portare una breve riflessione circa la reale efficacia dei diritti sociali nella salute pubblica brasiliana, utilizzando come base, un'analisi esplorativa di elucidações portato da vari studiosi che permeano a corroborare questo. Portando una breve discussione del minimo esistenziale.

1 Avvocato. Della medicina accademica. Dottorato di ricerca in sanità pubblica. Executive Mba. Specializzata in servizi sanitari applicata. Laurea in salute della famiglia. Specialista di audit. E-mail: autor@nucleodoconhecimento.com.br

2 Dottorato di ricerca in psicoanalisi, post dottorando in psicoanalisi clinica e master in bioetica, direttore del centro per la ricerca e lo sviluppo di studi avanzati, Coach e insegnante di corsi MBA in azienda, Campinas e Paulo São-e-mail: autor@nucleodoconhecimento.com.br

${ }^{3}$ Biologo. Master in biologia. Dottore in medicina tropicale/malattie. Ricercatore del programma del centro di medicina tropicale della UFPA laureato. Professore laureato livello e post-laurea e ricercatore presso I'Università federale del Pará - UFPA. E-mail: autor@nucleodoconhecimento.com.br 
Parole chiave: Diritti sociali. Sanità pubblica. Efficacia dei diritti fondamentali.

\section{INTRODUZIONE}

Diritti fondamentali possono essere definiti come un insieme di regole, principi, diritti, doveri e istituti coinvolti nella sovranità popolare, che garantiscono la convivenza pacifica, libero ed uguale, indipendentemente dal credo, razza, origine, colore, stato sociale o condizione economica, tutti basati sul principio della dignità umana. (CELLULE DEL SANGUE, 2010)

Tuttavia, a José Afonso da Silva, se concettualizzare diritti fondamentali diventa un compito difficile prima delle varie trasformazioni nel corso del tempo, vedere:

L'espansione e la trasformazione dei diritti umani fondamentali nella storia coinvolto rende difficile impostare loro un concetto sintetico.

Aumenta questa difficoltà la circostanza di impiegare diverse espressioni per designarli, come: diritti naturali, diritti umani, diritti umani, diritti individuali, diritti soggettivi pubblici, delle libertà fondamentali, le libertà civili e i diritti fondamentali e dei diritti umani fondamentali. (SILVA, 2005, p. 179)

Diritti sociali già, sono diritti fondamentali dell'uomo, come sta formulando Moraes (2014, p. 595):

I diritti sociali sono diritti fondamentali dell'uomo, perché si riferiscono ad un complesso di relazioni sociali, economiche o culturali che si sviluppa per la realizzazione della vita in tutte le sue potenzialità, senza che il titolare non poteva raggiungere e godere dei beni che avete bisogno.

Si scopre quindi che la principale difficoltà da affrontare in questo argomento si riferisce all'applicabilità e l'efficacia di questi diritti, perché come tutti sappiamo, la salute è positivada nell'elenco dei diritti sociali. 
Quindi, è importante qui menzionare il concetto del termine "efficacia" come il calcestruzzo prestazioni della funzione sociale del diritto, come segue:

La nozione di effettività, vale a dire, che specifica l'efficacia, corrisponde a quel Kelsendistinguere il concetto di efficacia di standard-interpretato come essere "la realtà del suo essere efficacemente attuata e osservato, il fatto che un essere umano condotta come standard per verificare i fatti". Efficacia significa la realizzazione del diritto, le prestazioni della sua funzione sociale. Lei rappresenta la materializzazione, nel mondo dei fatti, i precetti legali e simboleggia il riavvicinamento, quanto più vicino possibile, tra le normative e dovrebbe essere essere della realtà sociale. (BARROSO, 2002, p. 236)

Circa l'efficacia dei diritti sociali è necessario per la comprensione non solo dello stato di diritto in senso ampio, ma lo stesso approccio con altri sistemi, quali: politico, economico, sociale e storico.

È necessario anche la durata e l'efficacia della norma, come segue:

II normativismo distingue, con precisione, la durata dell'efficacia. La lezione di Kelsen è abbastanza chiara al riguardo. La durata dello standard, per lui, appartiene al mostoessere e non all'ordine di essere. Termine si intende che l'esistenza specifica lo standard; l'efficacia è il fatto che lo standard è efficacemente applicato e seguito; la circostanza che una condotta umana come la norma si applica ai fatti. (SILVA, 2005, p. 64)

Uno dei problemi più rilevanti che circonda l'efficacia dei diritti sociali fondamentali rispetto al modo sono stati organizzati nel testo costituzionale, considerato da molti autori e anche metodologicamente inadeguato. In questo senso, si afferma che:

Diritti sociali fondamentali nella costituzione brasiliana sono tutt'altro che formano un gruppo omogeneo, perché, per quanto riguarda il suo contenuto e il modo del suo riconoscimento, I'Assemblea costituente non ha seguito non costituito righe o teoria specifica. Invece, ha finito per creare un capitolo molto contraddittoria per quanto riguarda il rapporto interno di diritti e garanzie. Questo, senza dubbio, comporta una 
serie di inconvenienti interpretative, che colpiscono direttamente l'efficacia delle disposizioni contenute nella Costituzione. (KRELL, 2002, pag. 21)

Anche se, c'è questa confusione, per Ana Paula de Barcellos, l'efficacia giuridica della norma è direttamente collegata con il fundamentalidade sociale, come indicato di seguito:

II primo criterio che guida l'identificazione delle modalità di efficacia giuridica le dichiarazioni normative riguardanti è possono applicare uno stile di fundamentalidade di condizione sociale per lui governato, che è il tuo livello di importanza o rilevanza sociale. Questo è il parametro logico che guida la politica legislativa in generale. II più fondamentale per la società è materia disciplinata dal dispositivo e, di conseguenza, gli effetti che egli intende essere prodotto, più coerenza deve essere l'efficacia legale associato (...). (BARCELLOS, 2002, p. 136)

Di efficacia giuridica, capisce "capacità (potenziale) di una norma costituzionale a produrre effetti giuridici". (KRELL 2002, pag. 39).

Per produrre gli effetti desiderati di legali, l'efficacia giuridica dei diritti sociali, sono in piena efficacia norme suddividono, efficacemente contenuta e limitata efficacia, come spiegato di seguito.

Piena efficienza standard hanno applicabilità immediata e così sono indipendenti da legislazione successiva per la sua piena attuazione. Si afferma che:

Sono quelli che, dopo l'entrata in vigore della Costituzione, produrre, o possibilmente produrre tutti gli effetti essenziali, in relazione ai comportamenti, interessi, situazioni, che il legislatore costituente, diretto e normativamente, destinata a regolare. (SILVA, 2005, p. 101)

Norme costituzionali dell'efficacia già contenute sono che hanno carattere obbligatorio, ma sono anche limitazione del potere pubblico, come appare: 
Gli standard di efficienza contenuti sono quelli che il legislatore costituente ha regolato sufficientemente gli interessi relativi ad una questione particolare, ma in camera sinistra parte restrittiva della competenza discrezionale delle autorità pubbliche, in conformità con la legge che istituisce $o$ in termini di concetti generali in esso. (SILVA, 2005, pag. 116)

E ultimo ma non meno importante, gli standard di efficacia limitata che si basano sulla legge di regolamentarli. Sono chiamati anche "applicazione differita standard, standard di efficacia e l'efficienza standard su immediato" (cellule del sangue, 2010, p. 146)

Così si può vedere chiaramente che l'applicabilità dei diritti sociali è identico a quello delle garanzie e dei diritti fondamentali. Cioè, è applicabilità immediata come il § 1 dell'art. 5 di CF/1988.

\section{LA QUESTIONE DELL'EFFICACIA DEI DIRITTI SOCIALI NELLA COSTITUZIONE}

Un sacco di discutere la dottrina circa l'efficacia e l'applicabilità dei diritti costituzionali. Tuttavia, indipendentemente dalla forma di riconoscimento, gli standard dei diritti fondamentali devono generare un minimo di effetti giuridici, perché come si è visto, tutti gli standard ha l'efficacia e l'applicabilità.

Tuttavia, è che il grado di efficacia dei diritti sociali fondamentali sarà determinato dalla forma della Costituzione e il riconoscimento delle peculiarità del vostro oggetto. (SARLET, 2007, 237-238)

Il problema più grande, attualmente, non si riferiscono ai diritti, ma per la mancanza di efficacia delle norme costituzionali, vedere:

Il problema fondamentale in materia di diritti umani, oggi, non è tanto per giustificare la loro, ma per proteggerli (...). Non si tratta di una domanda di sapere cosa e come sono questi diritti, che la natura e la sua fondazione, che siano naturali o storico diritti, assoluto o relativo, ma che cosa è il modo più sicuro per garantire loro, per evitare che, 
nonostante le solenni dichiarazioni sono continuamente violati (BOBBIO, 1992, p. 2425)

In questo modo, l'efficacia ridotta del sociale fondamentale dei diritti non solo l'assenza di leggi ordinarie.

Il problema più grande non è la fornitura di servizi sociali di base da parte del governo, dal momento che la stragrande maggioranza delle norme per l'esercizio dei diritti sociali esiste già. Vedere se il problema è nella "formulazione, implementazione e manutenzione delle loro politiche pubbliche e nella composizione della spesa nei bilanci dell'Unione, gli Stati e i comuni". (KRELL, 2002, p. 31-32)

Un altro problema che si discute sulla dottrina è la determinazione degli elementi che costituiscono i diritti sociali fondamentali che si collega al fatto questi diritti esistono solo "quando le leggi e le politiche sociali la garanzia. In altre parole: è il legislatore ordinario che crea e determina il contenuto di un diritto sociale "(CANOTILHO, 2002, p. 481).

Questa notevole parte della dottrina ritiene che la maggior parte delle disposizioni costituzionali riguardanti diritti sociali "come incapace di presentare eventuali altre efficacia giuridica oltre a bind negativamente il legislatore da comportarsi in modo esplicito contro l'obiettivo indicato dalla norma". (BARCELLOS, 2002, p. 162)

Detto questo, sembra che gran parte della dottrina di solito classificare definizione di standard di diritti sociali come standard a livello di codice, poiché richiedono un successo legislativo che può generare la pienezza dei suoi effetti.

In questo senso, si afferma che a livello di codice standard sono:

Quelli nella legislatura costituente o non, invece di modificare la regola legale di applicazione pratica, solo tracce di amministrazione di linee, che guideranno le autorità pubbliche. La legislazione, l'implementazione e la giustizia sono soggette a questi dettami, che sono come i programmi dati alla funzione (BARROSO, 2002, p. 115) 
Ancora, secondo Krell (2002, p. 27-28), le norme programmatiche servono come alibi per creare un'immagine che lo stato risponde, normativamente, i problemi della società, vedere:

Molte norme costituzionali diritti sociali a livello di codice, per non avere un minimo di condizioni per la sua attuazione, servono solo un alibi per creare l'immagine di uno stato che risponde normativamente i problemi reali della società, giocando una funzione principalmente ideologica costituiscono una forma di manipolazione o illusione che Immunizza il sistema politico contro altre alternative.

Tuttavia, questo non sembra essere la tendenza attuale di effettività dei diritti sociali, nei confronti di tali argomenti, solo rivelare il pericolo se si lascia la volontà del legislatore o l'amministratore di determinare effetti sociali totali dei diritti fondamentali.

Come stati Barcellos (2002, p. 192): "ciò potrebbe causare un vero e proprio vuoto il fundamentabilidade del principio della dignità della persona umana come un vettore nel interpretazione costituzionale."

Va sottolineato qui, che la situazione diventa più complicata, dove il governo mantiene inerte, cioè dove non ancora installato i servizi necessari o dove lavorano male.

Gli effetti positivi dei diritti sociali dovrebbero essere forniti dallo stato e supportati dalla società, che sosterrà questi costi, raccolti attraverso le tasse tra gli altri come supportati:

Sicuramente ci sono risorse, più chiaro e precise forme testuali non sarà in grado di superare questa realtà fattuale, sarà impraticabile regole. Luís Roberto Barroso già identificare questa situazione, in cui l'apparente assenza di condizioni materiali condanna lo standard fin dalla sua nascita, come una forma di ipocrisia. Pertanto, se si desidera evidenziare, è che prendendosi cura dell'interpretazione del diritto pubblico in generale, e del trattato costituzionale particolare, è necessario avere in mente, oltre agli elementi puramente giuridici, dati della realtà, uno dei quali è le condizioni materiali e ottenere i controlli di regolamentazione finanziari. (BARCELLOS, 2002, p. 259-260) 
Tuttavia, prima che il malfunzionamento o anche la mancanza di servizi essenziali per il benessere della popolazione, "formulazione di politiche pubbliche di protezione di determinate categorie sociali economicamente emarginati ed esclusi", queste politiche che richiedono principalmente la gestione dello stato, essendo di fondamentale importanza per la sua attuazione, partenariati con la società civile. (KRELL, 2002, pag.

Presto, i diritti derivati ai benefici, derivanti da regolamenti di diritti sociali stabilite nella costituzione, implica, ove già distribuito il servizio pubblico necessario per la soddisfazione di un fondamentale diritto, che non la sua prestazione in violazione di legge ordinaria può, in Brasile, essere attaccato per mezzo di ingiunzione, strumento giuridico efficace il controllo giurisdizionale degli atti amministrativi.

Così, si afferma che:

L'efficacia che deve accompagnare le regole dei diritti sociali fondamentali soprattutto quando fondata sulla dignità della persona umana - deve essere positiva o simmetrica, perché consente la magistratura, se c'è una violazione incostituzionale, esecuzione di legge. Le modalità di interpretativa e vedativas efficacia negativa dell'inverso, anche se assolutamente rilevante e attuale, sono una protezione insufficiente di determinate variabili fattuali derivanti dall'imperativo del rispetto per il nucleo essenziale dei diritti sociali. (BARKER, 2011, p. 102-103)

Per questo motivo, per affrontare i problemi di effettività dei diritti sociali fondamentali, non c'è alcun modo per ignorare la loro funzione primaria dei diritti di prestazione, come pure il suo riconoscimento nel testo costituzionale, poiché entrambi gli aspetti, tutte le prove, non costituiscono fattori strettamente legate al grado di efficacia e applicabilità dei diritti fondamentali, come già accennato.

Un altro problema che mina l'efficacia dei diritti fondamentali è la cosiddetta riserva 'possibile', che è stata utilizzata per indicare la limitazione delle risorse prima che la necessità per la garanzia dei diritti fondamentali e sociali. Come dimostra Barcellos (2002, p. 261): 
II dibattito su questo tema è stato identificato in Brasile attraverso l'espressione possibile prenotazione e reso popolare in gran parte dal governo di impegno per promuoverlo e fa valere nelle esigenze più diverse, con il pretesto dell'imminente Apocalisse economica sempre.

Si scopre che la scarsità delle risorse di bilancio non può essere gli ostacoli per la garanzia dei diritti sociali, che sono condizioni essenziali della vita umana, perché in questo modo, in ultima analisi violare basilare e fondamentale precetto della Costituzione federale: il principio della dignità umana.

In questo modo, per garantire il minimo esistenziale è requisito necessario per la sopravvivenza dell'individuo, perché se non hai le condizioni necessarie per una vita dignitosa, le caratteristiche sociali non siano efficaci. E quando si tratta di minimo esistenziale, destinato per la salute, viola il diritto alla vita, perché senza la salute non si può vivere.

\section{IL PRINCIPIO DELLA PRENOTAZIONE POSSIBILE}

Non avete dubbio che la garanzia legale dei diritti sociali non dipendono solo i benefici positivi da parte dello stato, perché la loro efficacia dipende da risorse statali (Unione, stato, distretto federale e comuni), ma questi sono limitati. Per questo motivo, l'efficacia di questi diritti è limitata per le risorse di bilancio disponibili. Secondo Sarlet e boss (2007, p. 265):

Il posizionamento dei diritti sociali a prestazioni in virtù di quello che viene chiamato una possibile riserva che, inteso in senso ampio, che copre sia la possibilità, come il potere di disposizione da parte del destinatario della norma.

Per divagare sull'argomento, è necessario prima per concettualizzare il possibile riserva Nota:

La prenotazione di termine possibile tenta di identificare il fenomeno economico della limitazione delle risorse disponibili sulla necessità quasi sempre infinita per essere accolti da loro. Ciò che conta per lo studio intrapreso qui, i mezzi che, oltre alle 
discussioni legali su ciò che può essere previsto in tribunale dello stato - e l'ultima revisione della società, per esso è ciò che sostiene - è importante ricordare che c'è un limite di possibilità materiali per questi diritti. (BARCELLOS, 2002, p. 261-262)

Questa domanda circa l'effettiva disponibilità di fondi è visto dalla dottrina come una limitazione fattuale all'efficacia dei diritti sociali. Tuttavia, c'è anche un'altra limitazione che riguarda la possibilità legale per la disposizione delle risorse.

A proposito, ha detto Barcellos (2002, p. 262-263):

Sotto il titolo generale della riserva può vivere almeno due diverse specie di fenomeni. La prima di queste offerte con l'assenza di risorse fattuale, qualcosa vicino al bilancio e la stanchezza può essere identificata come una riserva di possibili fattuale. È possibile mettere in discussione la realtà di questo tipo di circostanza quando si tratta di enti pubblici, vista la forma di raccolta fondi e la natura dei biglietti. Comunque, la mancanza di risorse che descrivono situazioni su cui si potrebbe parlare di possibile fattuale riservati.

II secondo fenomeno identifica una riserva legale possibile poiché non descrive esattamente uno stato di esaurimento delle risorse e l'assenza di autorizzazione di bilancio spesa per alcuni in particolare.

Presto, all'inizio della riserva è una limitazione di fatto e di diritto che lo stato va per aiuto fornire diritti sociali a chi può interessare. Questo è il grosso problema qui sollevato, perché si riferisce alla richiesta più dal governo di questo principio, solo per evitare l'obbligo di attuazione dei diritti fondamentali.

In relazione a tale problema è indicato:

II Brasile ha una delle peggiori immagini della distribuzione del reddito nel mondo, e condizione la realizzazione dei diritti economici, sociali e culturali per l'esistenza di risorse disponibili nello stato, significa ridurre la sua efficacia a zero, mettere la loro universalità, condannandoli a considerare i diritti minori (KRELL, 2002, pag. 54) 
Ovvero, la maggior parte della popolazione brasiliana che è povera, cioè, carente nei servizi sociali è chi altro soffre di questo scontro di essere disporre l'esecuzione dei diritti sulla base della disponibilità delle risorse pubbliche.

Pertanto, anche se l'efficacia dei diritti sociali dipendono la considerazione del governo nelle loro scelte di fronte alla scarsità di risorse, lo stesso non può sottrarsi suo dovere costituzionale di fornire e garantire diritti minimi alla popolazione per una vita con dignità e in assenza di uno di essi, spetta la magistratura, attraverso la provocazione di risolvere qualsiasi problema sul tema e che si è verificato con la frequenza che mostra l'analisi del problema.

\section{CONSIDERAZIONI FINALI}

Supponendo che i bisogni della popolazione sono infiniti e limitate risorse, dovrebbe essere tenuto in mente su come stabilire le priorità nell'assegnazione delle risorse per garantire il minimo stabilito nella Costituzione federale.

In primo luogo, sottolineare che la formulazione del concetto minimo esistenziale ha provenuto la Corte costituzionale tedesca, che ha disegnato il diritto a un minimo di esistenza del principio della dignità umana (Costituzione, art. 1, I) e del diritto alla vita e all'integrità fisica, attraverso l'interpretazione sistematica del principio dello stato sociale (LF, arte. 20, IO) (KRELL.2002.

Integrando la comprensione:

Al primo, la qualificazione, qualificazione della dignità umana come un principio fondamentale che esprime la certezza che l'art. $1^{\circ}$, Inc. III, della nostra legge di base non contiene solo una dichiarazione di contenuti etici e morali (che ha, ma in ultima analisi, avere), ma che costituisce legale standard-positivo con status costituzionale e, come tale, con efficacia, trasformando tali fortunati, oltre la dimensione etica si è ricordata, valore giuridico della Comunità. In questo contesto, devono essere considerati, purché il principio fondamentale, la dignità della persona umana non è solo il valore di guida dei diritti fondamentali, ma dell'ordine intero costituzionale, che 
giustifica pienamente la sua caratterizzazione come un principio costituzionale della maggiore assiologica gerarchia-valutativa. (SARLET, 2007, p. 111-112)

Vale a dire il minimo esistenziale è legato alla dignità della persona umana e deve essere custodito dai diritti sociali caratteristiche al fine di fornire condizioni minime della popolazione.

Nel tentativo di delimitare il contenuto del minimo esistenziale, una possibile soluzione è l'uso del principio della dignità della persona umana per garantire le condizioni minime della popolazione per una vita sana ", suggerendo i parametri di linea guida come minimo stabilito dall'organizzazione mondiale della sanità. (SARLET, 2006, p. 47-59)

Eppure ci sono ancora grandi camera per la politica di un legislatore, poiché non vi è alcuna "una giusta misura o un modo unico di conformità con l'imperativo costituzionale", $\mathbb{E}:$ c'è un contenuto fisso del diritto ad un'esistenza dignitosa mínimopara, che può essere definita solo nel caso specifico. (ANDRADE, 2002, pag. 27)

Di tutti i tipi, il valore predefinito di minimo per la sopravvivenza deve sempre includere una base ed efficiente assistenza sanitaria, l'accesso al cibo base e abbigliamento, l'istruzione di primo grado e la garanzia di un'abitazione, come di seguito:

Ora, se la società non è in grado di riconoscere in ciò che punto le persone si trovano in una situazione indegna, cioè se non ci è consenso per quanto riguarda il contenuto minimo di dignità, "affronterà una crisi etica e morale di tali proporzioni che il principio della dignità umana si trasformerà in una formula totalmente vuota" (BARCELLOS 2002, p. 197)

Presto, il minimo sociale garantito nelle condizioni materiali di esistenza sarebbe basato sul concetto della dignità umana e la retorica di questo depreciaria minimo dei diritti sociali, ma piuttosto, rafforzerebbe nella loro dimensione essenziale come espressione della cittadinanza vendicativo. 
Costituzionalmente garantite, il minimo esistenziale deve essere riparato in via prioritaria, dice Barcellos (2002, p. 268):

Se è così e significa che le risorse finanziarie non sono illimitate, le risorse disponibili devono essere applicate in via prioritaria al servizio degli scopi considerati essenziali dalla costituzione fino al loro raggiungimento.

Le risorse rimanenti saranno progettati secondo le scelte politiche che deliberazione democratica per stabilire in ogni momento.

Considerando che il minimo esistenziale, inerente costituzionale garantisce priorità (caratteristiche dei diritti fondamentali), ha applicabilità immediata per essere stato nei diritti fondamentali indipendentemente dal fatto o meno sociale. D'altra parte, come la fornitura non sta trattando con i diritti sociali legati al minimo esistenziale, sarà condizionato le limitazioni delle risorse di bilancio.

Tuttavia, spetta alle autorità pubbliche il dovere di rispettare ciò che la costituzione garantisce come Krell (2002, pag. 60):

Dove lo stato crea queste offerte per il collettivo, deve garantire la possibilità di partecipazione dei cittadini. E se la legislazione non concede un diritto di esprimere singoli ricezione servizi vitali, il cittadino può fare appello al diritto fondamentale della parità in relazione al principio dello stato sociale

Una volta violato il minimo esistenziale, dall'omissione dell'efficacia dei diritti sociali e diritti, il diritto di richiedere in tribunale.

II motivo che giustifica tale giudiziarizzazione dei diritti sociali, inerenti al minimo esistenziale, in virtù della quale tali diritti sono indispensabili per avere una vita con dignità, che è la teoria della riserva non può presentare qui. Anche se giudiziarizzazione cercando di diminuire questo problema della mancanza di risorse, è necessario disporre di una vista che lo scopo essenziale prevista dalla Costituzione, e se le risorse sono sufficienti per soddisfare lo scopo previsto. (BARKER, 2011). 
Ma si trova ad affrontare il minimo esistenziale che valuterà le priorità per la spesa pubblica, sulla base della Costituzione e anche possibile definire l'attività giurisdizionale dei diritti sociali

Secondo Canotilho (2002), lo stato deve assicurare l'efficacia dei diritti sociali, come già sapete, ma vale a dire, troppo, che lo stesso non può ridurre, Annulla o revoca che cosa oggi capiamo come minimo esistenziale e deve essere sigillato tutte le misure legislative volte a questo. Questo è il principio del divieto del contraccolpo sociale.

Concettualizzare, se dice il contrario:

L'inverso, infine, è una derivazione dell'efficacia negativa, particolarmente legata ai principi che coinvolgono i diritti fondamentali. Lei si assume che tali principi sono attuati attraverso norme infra-costituzionale e che, basata sulla legge costituzionale vigente, un generale effetti previsto da tali principi è la progressiva espansione dei diritti fondamentali. A partire da questi presupposti, che l'inverso può richiedere la magistratura propone è l'invalidità la revoca di standard che, regolando il principio, per concedere o estendere i diritti fondamentali, senza il quale la revoca in questione è accompagnata da una politica di sostituto o equivalente. (BARROSO, 2002, p. 379)

Si conclude pertanto che questo sigillo è un modo per prevenire l'abrogazione delle leggi che garantiscono diritti sociali, non per sminuire ciò che è stato raggiunto attraverso l'evoluzione storica, come l'istituzione di norme minime al fine di avere una vita con dignità.

In questo modo, il ruolo della magistratura in materia di diritti sociali è importante perché è il controllo dei diritti sociali fondamentali, analizzando l'essenzialità del credito e il grado di bisogno secondo ogni singolo caso. E che questi judicialization di diritti sociali e soprattutto la salute, oggetto del presente studio, è chiamata la giudiziarizzazione del fenomeno di salute. 


\section{RIFERIMENTI BIBLIOGRAFICI}

ALMA-ATA. Alma Ata. 1978. Disponibile a: http://www.saudepublica.web.pt/05promocaosaude/Dec_Alma-Ata.htm. Accesso a: 09 nov. 2015.

ANDRADE, José Carlos Vieira de. II sistema giudiziario e diritti fondamentali sociali: accesso alla sanità, polemica di domanda. Porto Alegre: Corte di giustizia, 2002.

BARCELLOS, Emanuel. L'efficacia giuridica dei principi costituzionali: il principio della dignità umana. Rio de Janeiro: Renovar, 2002.

BARROSO, Luís Roberto. La legge costituzionale e l'efficacia delle sue regole: limiti e possibilità della costituzione brasiliana. 6 ed. São Paulo: Rinnovare, 2002

BARKER, Ramon F. Judicialization del diritto alla salute: la tensione tra il "minimo esistenziale" e la "riserva possibile" nella ricerca per la salvaguardia della dignità umana. Curitiba: Juruá Editora, 2011.

Brasile. Costituzione (1988). Costituzione della Repubblica federativa del Brasile. Brasilia. DF: Senato, 2014.

Brasile. Legge ${ }^{\circ} 8.080$ del 19 settembre 1990. Norme sulle condizioni per la promozione, la protezione e il recupero della salute, organizzazione e funzionamento dei servizi corrispondenti e altre questioni. Diário Oficial da União, ramo esecutivo, Brasília, DF.

CELLULE del sangue, Uadi Lammego. Diritto costituzionale alla portata di tutti. 2a ed. São Paulo: Saraiva, 2010.

CANOTILHO, José Joaquim Gomes. Diritto costituzionale e teoria della Costituzione. 6. ed. Coimbra: Almedina, 2002.

KRELL, Andrea Joachim. Diritti sociali e controllo giudiziario in Brasile e in Germania: i percorsi di (UN) da un diritto costituzionale comparato. Porto Alegre: Sergio Antonio Fabris, 2002 
Moraes, Guilherme peccato. Corso di diritto costituzionale. São Paulo: Atlas, 2014.

SARLET, Wolfgang Ingo. L'efficacia dei diritti fondamentali. 7. Ed. Porto Alegre: Editora avvocato, 2007.

. Dignità umana e i diritti fondamentali nella Costituzione federale del 1988. 4 ed. Porto Alegre: Editora avvocato, 2006.

José Afonso da SILVA. Contestuale commento sulla Costituzione. São Paulo: Malheiros, 2005. 